\title{
Hyperspectral Image Reconstruction Using Multi-colour and Time-Multiplexed LED Illumination
}

5

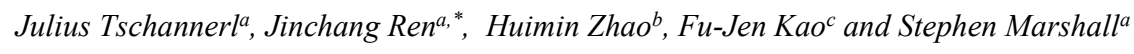

${ }^{a}$ Centre for Signal and Image Processing, University of Strathclyde, Glasgow, UK

${ }^{b}$ School of Computer Science, Guangdong Polytechnic Normal University, Guangzhou, China

${ }^{c}$ Institute of Biophotonics, National Yang-Ming University, Taipei, Taiwan, R.O.C

${ }^{*}$ Corresponding author: jinchang.ren@strath.ac.uk

\begin{abstract}
The rapidly rising industrial interest in hyperspectral imaging (HSI) has generated an increased demand for cost effective HSI devices. We are demonstrating a mobile and low-cost multispectral imaging system, enabled by time-multiplexed RGB Light Emitting Diodes (LED) illumination, which operates at video framerate. Critically, a deep Multi-Layer Perceptron (MLP) with HSI prior in the spectral range of $400-950$ $\mathrm{nm}$ is trained to reconstruct HSI data. We incorporate regularisation and dropout to compensate for overfitting in the largely ill-posed problem of reconstructing the HSI data. The MLP is characterised by a relatively simple design and low computational burden. Experimental results on 51 objects of various references and naturally occurring materials show the effectiveness of this approach in terms of reconstruction error and classification accuracy. We were also able to show that utilising additional colour channels to the three R, G and B channels adds increased value to the reconstruction. Keywords: Hyperspectral imaging (HSI), deep learning, spectral reconstruction, LED illumination
\end{abstract}

\section{Introduction}

In addition to the traditional applications of Hyperspectral Imaging (HSI) in remote sensing [1-3], the recent and rapid rise of interest for HSI was focused on various industrial applications, such as food quality monitoring [4-6], counterfeit detection [7] 
and artwork authentication [8,9]. Conventional HSI systems are, however, very cost intensive and require relatively complicated operation. Line scanning for HSI, frequently referred to as pushbrooming, is characterised by high spectral resolution due to the use of dispersive elements that split the incident light of a scanned line into hundreds of very narrow bands. Pushbrooming requires a linear translation of either the camera or the scanned objects, thus resulting in a lower spatial resolution. In contrast, area scanning is characterised by high spatial and low spectral resolution, determined by the number of filters utilised, and requires the objects to be static. Both technologies suffer from time consuming scanning processes and require careful operation to preserve geometric accuracy and minimise spectral smearing. A third approach that has overcome the issue of long integration period and found increasing interest in the last years is snapshot HSI. Recent advances in compressive sensing have led to technologies such as coded aperture [10], which even allows for Raman snapshot HSI [11]. Snapshot imaging, however, also suffers frequently from the requirement of very costly high precision optical components, as well as reduced spectral and spatial resolution and overwhelming amounts of data in a short timespan [12].

A recent and promising focus is the approximation of HSI data from Red, Green and Blue (RGB) or multispectral imaging (MSI). Light Emitting Diode (LED) technology is a very powerful and low-cost technology that is increasingly interesting for the development of multispectral devices [13]. Goel et al. [14] have proposed an MSI system that utilises a set of LEDs with 17 distinct peak wavelengths in the range of 450 $-990 \mathrm{~nm}$ to realise time-multiplexed illumination, which shows good results in various applications. The use of LEDs makes the system very cost effective and flexible, however, the selection of peak wavelengths is hard-wired and lacks a generalised solution. Herrera-Ramirez et al. [15] proposes an LED based system with a considerable broader spectral range of $370-1630 \mathrm{~nm}$, in which interpolation is used to recover hyperspectral information from 22 wavelengths. While the system is portable and lowcost, interpolation runs the risk of overlooking specific spectral absorption peaks of 60 materials not covered by the illumination source. Hyperspectral recovery from mere RGB images was proposed by Arad et al. [16], in which a sparse dictionary is trained on a database of hyperspectral prior. By matching CIE colour coordinates, a transformed RGB dictionary is generated to reconstruct the original HSI information 
from the RGB images. The system is, however, limited to RGB images and requires prior knowledge regarding the spectral sensitivity of the camera system, which is usually not available. Inspired by the concept of spatial super-resolution, Galliani et al. [17] have proposed a reconstruction algorithm from RGB images based on deep Convolutional Neural Networks (CNN). Can et al. [18] improved the reconstruction results by designing a simplified CNN with fewer layers which is less prone to overfitting and computationally less expensive. Recent developments in deep learning focus enable CNNs to be equally less prone to label noise [19] and the introduction of new architectures significantly reduces training complexity [20]. Nonetheless, CNNs evolve with an increasing complexity with respect to their design and often require GPUs to accommodate the excessive computation. Designing shallower networks can reduce the amount of GPU memory required, which, nevertheless, still poses a significant limit on the design.

In this work, we are proposing an MSI system that is based on time-multiplexed RGB LED illumination. The advantages of the system are low-cost, high mobility, high framerate, and improved efficiency in hyperspectral data reconstruction. By mapping the collected MSI data to HSI prior to train a relatively shallow neural network, we aim to realise a computationally efficient algorithm for HSI data reconstruction. This work extends our presentation at the Hyperspectral Imaging and Applications Conference 2018 [21]. This includes a more robust reconstruction algorithm as well as an extended evaluation of the reconstructed spectra for reference as well as organic materials with respect to both reconstruction error and classification accuracy.

The main contributions of this paper entail the following: The proposed multichannel camera system poses a novel rapid, portable and low-cost imaging technique for effective data acquisition. A neural network based reconstruction proves the feasibility of using multi-colour data for hyperspectral reconstruction. It is also shown that using additional colour channels to common red, green and blue add valuable information and decrease noise. 


\section{Proposed System}

The proposed system comprises of two main components. At first, multi-colour and hyperspectral data of the same objects are acquired. This is used to train a reconstruction algorithm, that is then able to reconstruct hyperspectral data from unknown input containing objects with the same spectral characteristics. This is visualized in Figure 1. The individual components are detailed in this section.

\subsection{Hardware architecture}

The architecture of the proposed system is schematically represented in Figure 2. It comprises a Basler ace $U$ monochromatic camera with a spatial pixel resolution of 1280 x 1024 pixel (HD), a framerate up to 200 fps and a USB 3.0 interface. The image integration is triggered by an Arduino Uno, which also controls an Adafruit Neopixel ring containing $24 \mathrm{WS} 2812 \mathrm{~B}$ based RBG LEDs. The LEDs are programmed to produce eleven different colours in sequence and the image acquisition is triggered by the switch of colour, realising time-multiplexed illumination. The image data from the camera is recorded by the Single Board Computer Odroid X4U. Depending on the number of colour channels used, this allows for a recording speed from 18 (eleven channels) up to 200 (one channel) images per second, which allows the system to operate in video framerate. Notably, the components amount to a cost less than GBP 1000.

The selected eleven colours were chosen to emulate wavelengths between $400-650$ $\mathrm{nm}$ using colour emulation that match the CIE 1931 standard observer. The colours were selected to be approximately equally spaced at $25 \mathrm{~nm}$ in terms of wavelength in that region. The settings for the intensities of the three colour channels are illustrated in Figure 3. Note that RGB LEDs can only generate colours by mixing intensities of the three RGB lights with very distinct peak wavelengths at 465 - $467 \mathrm{~nm}$ (B), $522-525$ $\mathrm{nm}(\mathrm{G})$ and 620 - $625 \mathrm{~nm}(\mathrm{R})$ [22]. The irradiance of the generated colours was measured with a spectroscope and the results are shown in Figure 3. It is apparent that the peak wavelengths remain the same, while the intensity combinations vary. We believe that varying the illumination colour (or intensity combinations), even without 
generating new wavelengths, increases variability within the data and therefore the accuracy of the predictive system.

\subsection{Hyperspectral reconstruction}

The reconstruction is performed by training a Multi-Layer Perceptron (MLP) defined as follows. An input matrix $\mathbf{X}=\left\{\mathbf{x}_{1}, \mathbf{x}_{2}, \ldots, \mathbf{x}_{n}\right\}^{T}$ of the MSI vectors $\mathbf{x}_{i}$ is mapped to an output matrix, $\mathbf{Y}=\left\{\mathbf{y}_{1}, \mathbf{y}_{2}, \ldots, \mathbf{y}_{k}\right\}^{T}$, where $k$ is the number of objects and $n$ is the number of MSI observations per object. The MLP consists of four fully connected (FC) hidden layers $\mathbf{x}_{i+1}=\sigma_{i}\left(\mathbf{W}_{i} \mathbf{x}_{i}+\mathbf{b}_{i}\right)$ with activation function $\sigma_{i}$, weight matrix $\mathbf{W}_{i}$ and bias $\mathbf{b}_{i}$. The detailed architecture is visualised in Figure 4 . The fact that the input layer has no activation function, i.e. $\sigma_{1}=f(\mathbf{x})=\mathbf{x}$ enforces the use of all MSI bands to further reduce fluctuations. For training, we define a loss function $\mathcal{J}(\theta)$ of the parameter vector $\theta=\left\{\mathbf{W}_{1}, \ldots, \mathbf{W}_{i}, \mathbf{b}_{1}, \ldots, \mathbf{b}_{i}\right\}, i \in 1, \ldots 5$. For the predicted output $\widehat{\mathbf{Y}}$, a simple loss function can be defined as:

$$
\mathcal{J}(\theta)=\frac{1}{2 n}\|\widehat{\mathbf{Y}}-\mathbf{Y}\|_{F}
$$

where $\|\cdot\|_{F}$ denotes the Frobenius norm. The problem, however, is highly ill-posed, as the input dimensionality is significantly lower than the output dimensionality and slight changes in the input can translate into a severe alteration at the output. Regularisation is a commonly used practice to compensate for these effects [23]. Introducing soft constraints on the parameters of the MLP can also help reduce the validation error and promote generalisation. Typically, regularisation is only applied onto the weights of the MLP [23], since the biases require less data to be fitted and regularising both may lead to significant underfitting. As the most common regularisation term, we introduce $L^{2}$ Norm regularisation on the weight, often referred to as weight decay, with hyperparameter $\beta$ that controls the impact of the regularisation:

$$
\mathcal{J}(\theta)=\frac{1}{2 n}\|\widehat{\mathbf{Y}}-\mathbf{Y}\|_{F}+\frac{\beta}{2} \sum_{i=1}^{5}\left\|\mathbf{W}_{i}\right\|_{F}
$$

For further regularisation, dropout is introduced here. Dropout is a computationally inexpensive way of approximating bagging, which describes the process of combining the results of several separately trained models [23]. It is achieved by effectively 
generating a binary mask of randomly sampled values multiplied with a non-output layer to remove certain nodes. The probability at which nodes are kept is called the keep rate and is another hyperparameter of the network. Dropout roughly doubles the number of iterations required for convergence [24].

\subsection{Implementation details}

The implementation was done in the Tensorflow framework for Python, using the Adam Optimiser with the initial learning rate of 0.001 . $\beta$ was set to $10^{-6}$ and a batch size of 128 was used for training. A keep rate of 0.6 for the dropout was used and in accordance with the definition of the popular AlexNet [24] only applied on the last hidden layer. Weight decay and dropout both minimise effects of overfitting and consequently enforce a relatively smooth output which satisfies smoothness characteristics of hyperspectral data. For optimally smooth results, we established empirically that 5,000,000 iterations are necessary.

\section{Experimental results}

To verify the system, the proposed camera was used to image 20 differently coloured fabrics such as cotton, wool and polyester in various shades including four different blacks, four very bright materials in white, grey, cream and bright yellow, two blue shades, turquoise and two green shades and seven different red, orange and pink shades. These fabrics were chosen to test for the discriminability between subtle changes in colour. Additionally, the Macbeth ColorChecker chart with 24 colour patches and seven different organic objects, including red and white grapes, bananas, apples, pears, spinach and tomatoes were imaged to include some naturally occurring materials. The imaged objects are illustrated in Figure 6. In total, 51 different objects were imaged with an HSI system covering the visible near infrared (VNIR) range from $400-950 \mathrm{~nm}$ in 256 bands. For each class, the mean spectrum was calculated and used to train the MLP. The same objects were imaged with the proposed MSI system, and equally 4000 measurements were used to train and evaluate the system. The MSI images were acquired in a dark room to minimise the effects of ambient lighting. For production use 
of the system, an ambient light image can be acquired and subtracted from all successive images to realise calibration. Both datasets were radiometrically calibrated and converted to reflectance using white and dark reference images. In theory, the MSI system only needs to be calibrated once, as the illumination is always constant. Given the influence of ambient light and the variable sensor sensitivity and LED radiance, it is still advisable to repeat the calibration before every imaging session.

In the first experiment, we tried to analyse the effect different numbers of bands have on the quality of reconstruction. The results for the Root Mean Squared Error (RMSE) achieved after 1,000,000 iterations with 5 repetitions are shown in Figure 5. The bands were chosen to be as equally spaced as possible out of the eleven totally recorded bands. Interestingly, it can be seen that at least 6 colour channels are required to minimise the reconstruction error. This leads us to the conclusion that adding more colour channels adds valuable information to the reconstruction. According to these results, we have compared the reconstructed spectrum profiles from three, six and eleven bands respectively in additional experiments, as both six and eleven channels produce an equally low RMSE but recording less bands also potentially increases the framerate of imaging. Both were compared with three channel reconstruction to highlight the additional information gain over RGB. The three channels that are closest to RGB were selected to highlight the information gain over plain RGB images.

For quantitative evaluation, the 51 imaged objects were grouped in three groups; coloured fabrics, organic materials, and Macbeth chart patches. The reconstruction was evaluated using three different measures, The RMSE, Spectral Angular Mapper (SAM) and Spectral Information Divergence (SID) [25]. SAM is similar to the cosine distance as it measures the angle between spectra in an n-dimensional space and is therefore invariant to scaling. SID, in contrast, measures similarity by modelling the spectra as probability distributions, using information theory. A high SID value indicates great similarity. Reconstruction results after 5,000,000 iterations with three, six and eleven channels are given in Error! Reference source not found.. The reconstruction using eleven bands performs generally the best. The coloured fabrics produces a higher similarity to the original HSI data, whereas both the fruit and vegetable and the Macbeth chart generate very similar errors for both six and eleven channels. This shows that the fabrics can be reconstructed very accurately, even though some of them only vary 
slightly in the shade of the respective colour. What we can also observe is that both six and eleven channels produce a significantly lower reconstruction error than the three plain RGB channels. This leads to the conclusion that adding more colour channels reduces noise and likely adds new information to the MLP and therefore improves the reconstruction significantly.

\begin{tabular}{|c|c|c|c|}
\hline & Fabric & Organic & Macbeth chart \\
\hline \multicolumn{4}{|c|}{3 Channels } \\
\hline RMSE & $0.475 \pm 0.500$ & $0.947 \pm 0.630$ & $1.279 \pm 0.279$ \\
\hline$S A M$ & $0.077 \pm 0.028$ & $0.114 \pm 0.053$ & $0.122 \pm 0.011$ \\
\hline$S I D$ & $14.808 \pm 4.60$ & $0.858 \pm 0.386$ & $0.435 \pm 0.038$ \\
\hline \multicolumn{4}{|c|}{6 Channels } \\
\hline RMSE & $0.277 \pm 0.218$ & $0.439 \pm 0.211$ & $0.502 \pm 0.038$ \\
\hline$S A M$ & $0.065 \pm 0.019$ & $0.064 \pm 0.021$ & $0.075 \pm 0.004$ \\
\hline$S I D$ & $15.60 \pm 3.420$ & $3.594 \pm 1.317$ & $2.412 \pm 0.405$ \\
\hline \multicolumn{4}{|c|}{11 Channels } \\
\hline RMSE & $0.128 \pm 0.095$ & $0.228 \pm 0.143$ & $0.219 \pm 0.032$ \\
\hline$S A M$ & $0.032 \pm 0.015$ & $0.034 \pm 0.013$ & $0.046 \pm 0.003$ \\
\hline$S I D$ & $20.14 \pm 3.436$ & $5.553 \pm 1.732$ & $3.508 \pm 2.912$ \\
\hline
\end{tabular}

Table 1: Reconstruction errors for the object groups. For 3 channels, channels 2, 6 and 11 were used according to Figure 3 and for 6 channels these are 1, 3, 5, 7, 9 and 11 .

The reconstruction is visualised in Figure 6. Only representative classes of objects were chosen that display certain attributes of the reconstruction. The coloured fabrics have relatively distinct spectral signatures, due to differences in material and colouring, and thus can be quite accurately reconstructed. For black fabrics, the initially low signals lead to a less accurate reconstruction, as shown by a less smooth curve. The linen and the polyester display almost exactly the same spectral characteristics in the spectral coverage of the illumination, despite their differences in the spectral range from $700 \mathrm{~nm}$ upwards. our camera system is likely not to pick up on these features as the illumination does not cover this spectral range. Consequently, the reconstructed spectra of these two classes of objects are almost identical. The metrical differences to the 
original spectra are however still small, which leads to the high similarity in Error!

\section{Reference source not found.}

For the fruit and vegetable, the input MSI data is more varied as the surfaces of the scanned materials are not homogeneous. As a result, the reconstruction shows some deviation from the original spectra but still adheres well to the HSI data. Specific features, such as the typical spike in reflectance from $680-730 \mathrm{~nm}$ caused by chlorophyll and referred to as the red-edge, is retained very well for the spinach.

For the Macbeth chart, the colours show very distinct spectral reflectance and can therefore be reconstructed very accurately, reflecting the nature and the usage of the chart. Difficulties arise for the grey (neutral) and black patches, where the low intensity of the signal and their flatness leads to errors in the reconstruction.

To further validate the quality of reconstruction, a Support Vector Machine (SVM) with a radial basis function (RBF) was trained to classify all 51 classes using 600 samples of each object and $5 \%$ of each class trained and the rest to validate. The parameters $C$ and $\gamma$ of the SVM were trained using a grid search with 5-fold cross validation. The 51 classes were again grouped and the mean pixel-wise overall accuracy (OA) was calculated for each group and summarised in Table $\mathbf{2}$.

\begin{tabular}{lllll}
\hline & Original & 3 Channels & 6 Channels & 11 Channels \\
\hline Fabrics & $98.80 \pm 2.89$ & $86.84 \pm 4.88$ & $91.89 \pm 3.28$ & $93.10 \pm 2.92$ \\
Organic & $99.81 \pm 0.63$ & $91.81 \pm 1.78$ & $98.62 \pm 0.65$ & $98.86 \pm 0.57$ \\
Macbeth chart & $98.49 \pm 2.89$ & $98.55 \pm 0.63$ & $99.92 \pm 0.08$ & $99.91 \pm 0.14$ \\
\hline OA & $98.79 \pm 2.58$ & $93.22 \pm 2.45$ & $96.59 \pm 1.41$ & $97.45 \pm 1.29$ \\
\hline
\end{tabular}

Table 2: Comparison of classification using the original 256 bands; the reconstruction of these bands from 3, 6 and 11 channels. For 3 channels, channels 2, 6 and 11 were used according to Figure 3 and for 6 channels these were 1, 3, 5, 7, 9 and 11.

SVMs were trained on the original 256 band HSI data and the reconstructed spectra using three, six and eleven channels from the MSI system. According to Table 2, we can again see that the reconstruction using eleven channels generates better results than the six-channel one. Specifically, the fabrics show the largest difference, $98.80 \%$ with the original data versus $86.84 \%$ with three channels, $91.89 \%$ with six-channel, and 
$93.10 \%$ with eleven-channel. For the organic materials, the classification accuracy is almost the same for six and eleven channels but almost $7 \%$ lower with three channels. For the Macbeth chart, the classification seems to improve only by a small margin, even with three channels, a very high accuracy can be achieved. The decrease in classification accuracy for fabrics is likely attributed to the reduced intensity, as mentioned above, in the black fabrics. Even though the reconstructions are very close to the originals, they are almost identical to each other. Consequently, a low reconstruction error is achieved despite the decreased classification accuracy. When adding additional channels, subtler variances can be detected and signals with a low SNR can be better reconstructed. Likewise, for the organic materials, the surface of the objects is subject to more variation and simple RGB colour sensing does not seem to account for all the differences in the signals measured with an HSI system. For the Macbeth chart however, we see that the three RGB channels produce an almost identical classification after HSI reconstruction, which is likely due to the fact that the chart itself is designed to calibrate RGB imaging systems and therefore only displays spectra that are produced by primary colours. Using three RGB channels accounts for almost all variances, where adding additional channel only helps marginally improving the classification accuracy.

\section{Conclusion}

In conclusion, we have shown that customary and adaptive RGB LED illumination can be integrated with a monochromatic camera to achieve a very cost efficient and mobile MSI camera system. The training of a neural network that maps MSI data to HSI prior is conducted by reconstructing hyperspectral profiles of different objects. Despite RGB LEDs having only three distinct peak wavelengths, mixing them with various intensities allows the generation of sufficient variability to accurately reconstruct the HSI data and therefore adds additional information to the predictive system compared to only three-channel RGB illumination. The physical advantages in using RGB LED technology include its availability and maturity, cost effectiveness, and flexible configuration. Additionally, LED allows ring-shaped (or other 
arrangement) illumination that enables uniform lighting for each colour channel with no additional measures to eliminate artefacts such as chromatic shadows that are caused by angular non-uniformity. For optimal reconstruction, eleven channels were used with a framerate of 18 MSIs per second and a high spatial pixel resolution. The integration rate can be further increased by sacrificing some of the reconstruction quality. It is also shown that a reconstruction with six channels and a framerate of 33 MSIs per second still generate very good results. The reconstruction is tested on 3 groups of objects, as detailed in the experimental results. It shows that a highly accurate reconstruction is possible when the original HSI spectra are sufficiently distinct. Errors occur when the measured signal is low in intensity and with high similarity in the spectral range (400$650 \mathrm{~nm}$ ) covered by the RGB LEDs, regardless of the differences in the spectral region outside. A remedy or improvement is to increase LEDs to cover a broader spectral range. Due to the nature of supervised machine learning, this system is reliant on HSI prior and requires pre-training on spectral signatures of imaged objects to be functional. Given that many HSI applications are very specific and limited in the number of occurring spectra, this poses not a major disadvantage.

Importantly, this prototype system promises a cost effective and mobile hyperspectral imaging setup with a high spatial pixel resolution and framerate. The setup is flexible in configuration and can be trained for various applications. Future work will further improve the illumination design to cover broader spectral peaks, especially the range above $650 \mathrm{~nm}$, to enable more applications. Additionally, designing LED illumination with distinct spectral peaks could potentially improve the reconstruction. Algorithms for hyperspectral band selection [26,27] can help identify relevant spectral bands to design such illumination systems.

\section{References}

[1] Young A, Marshall S, Gray A. Outlier and target detection in aerial hyperspectral imagery: a comparison of traditional and percentage occupancy hit or miss transform techniques. Proc. - Autom. Target Recognit. XXVI, Baltimore, Maryland, United States: 2016, p. 98440 S. 
doi: $10.1117 / 12.2213530$.

[2] Alam MS, Islam MN, Bal A, Karim MA. Hyperspectral target detection using Gaussian filter and post-processing. Opt Lasers Eng 2008;46:817-22. doi:10.1016/j.optlaseng.2008.05.019.

[10] Lin X, Wetzstein G, Liu Y, Dai Q. Dual-coded compressive hyperspectral imaging. Opt Lett 2014;39:2044. doi:10.1364/OL.39.002044.

[11] Thompson J V., Bixler JN, Hokr BH, Noojin GD, Scully MO, Yakovlev V V. 
Single-shot chemical detection and identification with compressed hyperspectral Raman imaging. Opt Lett 2017;42:2169. doi:10.1364/OL.42.002169.

[12] Hagen N, Kudenov MW. Review of snapshot spectral imaging technologies. Opt Eng 2013;52:090901. doi:10.1117/1.OE.52.9.090901.

[13] Bian Y, Liu Q, Zhang Z, Liu D, Hussian A, Kuang C, et al. Portable multispectral lens-less microscope with wavelength-self-calibrating imaging sensor. Opt Lasers Eng 2018;111:25-33. doi:10.1016/j.optlaseng.2018.07.009.

[14] Goel M, Patel SN, Whitmire E, Mariakakis A, Saponas TS, Joshi N, et al. HyperCam: Hyperspectral Imaging for Ubiquitous Computing Applicaitons. Proc. 2015 ACM Int. Jt. Conf. Pervasive Ubiquitous Comput. - UbiComp '15, New York, New York, USA: ACM Press; 2015, p. 145-56. doi:10.1145/2750858.2804282.

[15] Herrera-Ramírez J, Vilaseca M, Pujol J. Portable multispectral imaging system based on light-emitting diodes for spectral recovery from 370 to $1630 \mathrm{~nm}$. Appl Opt 2014;53:3131. doi:10.1364/AO.53.003131.

[16] Arad B, Ben-Shahar O. Sparse Recovery of Hyperspectral Signal from Natural RGB Images. Proc. - 14th Eur. Conf. Comput. Vis. (ECCV '16), 2016, p. 1934. doi:10.1007/978-3-319-46478-7_2.

[17] Galliani S, Lanaras C, Marmanis D, Baltsavias E, Schindler K. Learned Spectral Super-Resolution. CoRR 2017.

370 [18] Can YB, Timofte R. An efficient CNN for spectral reconstruction from RGB images. CoRR 2018.

[19] Ding G, Guo Y, Chen K, Chu C, Han J, Dai Q. DECODE: Deep Confidence Network for Robust Image Classification. IEEE Trans Image Process 2019:11. doi:10.1109/TIP.2019.2902115. Trans Image Process 2018;27:4357-66. doi:10.1109/tip.2018.2835143. 
[21] Tschannerl J, Ren J, Marshall S. Low Cost Hyperspectral Imaging Using Deep Learning Based Spectral Reconstruction. Proc. - Hyperspectral Imaging Appl. Conf. (HSI 2018), 2018.

2018.

https://cdnshop.adafruit.com/datasheets/WS2812B.pdf (accessed September 7, 2018).

[23] Goodfellow I, Bengio Y, Courville A. Deep Learning. MIT Press; 2016.

[24] Krizhevsky A, Sutskever I, Hinton GE. ImageNet Classification with Deep Convolutional Neural Networks. Proc. - Adv. Neural Inf. Process. Syst. (NIPS 2012), 2012, p. 1097-105.

[25] Chein-I Chang. Spectral information divergence for hyperspectral image analysis. IEEE 1999 Int. Geosci. Remote Sens. Symp. (IGARSS’ 99), vol. 1, IEEE; 1999, p. 509-11. doi:10.1109/IGARSS.1999.773549.

[26] Cao X, Wei C, Han J, Jiao L. Hyperspectral Band Selection Using Improved Classification Map. IEEE Geosci Remote Sens Lett 2017;14:2147-51. doi:10.1109/LGRS.2017.2755541.

[27] Jiao L, Han J, Cao X, Ji B, Wang L, Ji Y. Fast hyperspectral band selection based on spatial feature extraction. J Real-Time Image Process 2018;15:55564. doi:10.1007/s11554-018-0777-9. 